\title{
Three-dimensional analysis of seminiferous tubules and spermatogenic waves in mice
}

\author{
Hiroki Nakata ${ }^{1}$, Takahiro Sonomura ${ }^{2,3}$ and Shoichi Iseki ${ }^{1}$ \\ ${ }^{1}$ Department of Histology and Cell Biology, Graduate School of Medical Sciences, Kanazawa University, \\ Takaramachi, Kanazawa, Japan, ²Department of Anatomy II, School of Medicine, Kanazawa Medical University, \\ Uchinada, Ishikawa, Japan and ${ }^{3}$ Department of Oral Anatomy, Asahi University School of Dentistry, Mizuho, Japan
}

Correspondence should be addressed to H Nakata; Email: hnakata@staff.kanazawa-u.ac.jp

\begin{abstract}
The aim of the present study was to reconstruct seminiferous tubules and analyze spermatogenic waves in seminiferous epithelia in developing and adult mice using serial paraffin sections and high-performance three-dimensional (3D) reconstruction software. By labeling the basement membrane of seminiferous tubules with fluorescent immunohistochemistry or periodic acid-Schiff-hematoxylin staining, all seminiferous tubules were reconstructed in 9 testes from 9 different mice, 3 each at 0, 21 and 90 days (adult) postpartum. The 3D structure of seminiferous tubules, including the number and length of tubules as well as the number of connections with the rete testis, branching points and blind ends, was assessed accurately. Although tubules showed marked variations among individual mice, their overall structure was regular and retained from newborn to adult mice. Some seminiferous tubules contained inner portions running distant from the testis surface. In a representative testis at 21 days, the sites at which spermatids initially occurred were examined by labeling acrosomes and were found to be preferentially distributed in the upper and medial portions of the testis close to the rete testis. In a representative adult testis, 76 complete waves with an average length of $16.9 \mathrm{~mm}$ were found and their directions were analyzed. The methods used in the present study will be useful for investigating the structure and function of seminiferous tubules in mice and humans under normal and pathological conditions, such as infertility.

Reproduction (2017) 154 569-579
\end{abstract}

\section{Introduction}

Spermatogenesis is the process of cellular transition from undifferentiated spermatogonia to mature spermatozoa in the seminiferous tubules. The seminiferous epithelium is divided into 14 stages in rats and 12 stages in mice according to the cellular associations in a given area (Leblond \& Clermont 1952, Oakberg 1956, Ahmed \& de Rooij 2009, Meistrich \& Hess 2013, Nakata et al. 2015a). Adjacent stages are aligned along a seminiferous tubule, and the time required for a particular stage to reappear in the same area is called the cycle (Leblong \& Clermont 1952), whereas the space occupied by a series of adjacent stages including all possible types is called the wave (Perey et al. 1961). Based on accurate information on stages, the morphological features of the seminiferous epithelium in many genetically modified and mutant mouse strains with male infertility have been analyzed using testis sections (de Rooij \& de Boer 2003, Matzuk \& Lamb 2008). However, the precise threedimensional (3D) structure of seminiferous tubules is not easy to assess by observing the testis sections.

Curtis (1918) was the pioneer for using serial sections of the testis for the 3D reconstruction of mammalian seminiferous tubules. He reconstructed two complete seminiferous tubules from an adult mouse testis and estimated that one testis contained 15 seminiferous tubules with a few branching points. Clermont and Huckins (1961) reconstructed 20 seminiferous tubules in an adult rat testis using serial sections and found three branching points and one blind end. They described two types of seminiferous tubules: outer tubules that formed funnel-shaped networks and were located close to the tunica albuginea and inner tubules that formed cone-shaped networks and were located far from the tunica albuginea. Since all reconstruction steps were conducted manually, it was not until recently that any additional information was obtained on the $3 \mathrm{D}$ structure of adult rodent seminiferous tubules.

Our group recently reported the high-resolution 3D structure of all seminiferous tubules in an adult mouse testis using serial sections and high-performance 3D reconstruction software (Nakata et al. 2015b). We found 11 seminiferous tubules, 28 connections with the rete testis, 9 branching points and one blind end in one testis. Since all seminiferous tubules had portions close to the tunica albuginea, we were unable to distinguish the two types of seminiferous tubules described for the rat testis by Clermont and Huckins (1961). The method 
adopted in our previous study required the manual segmentation of seminiferous tubule outlines, and, thus, was accurate, but laborious and time-consuming. The use of immunofluorescence staining of the basement membrane was expected to make the segmentation of seminiferous tubules semi-automatic and save time. Furthermore, the use of fluorescence lectin staining of the acrosome (Nakata et al. 2015a, 2017) was expected to provide additional information on waves in the seminiferous epithelium.

In the present study, we intended to 3D-reconstruct seminiferous tubules in developing and adult mice and analyze spermatogenic waves in seminiferous epithelia in reconstructed tubules using the semi-automatic segmentation method.

\section{Materials and methods}

\section{Animals}

The present animal study was specifically approved by Kanazawa University (approval number: AP-153636) and conducted in accordance with the Guidelines for the Care and Use of Laboratory Animals in Kanazawa University. C57BL/6 strain male mice were purchased from Nippon SLC, Inc. (Hamamatsu, Japan), reared under standard 12-h light/12-h darkness laboratory conditions with free access to standard food and water and used within $24 \mathrm{~h}$ of birth (P0), 21 days after birth (P21) and 90 days after birth (P90 or adult).

\section{Tissue preparation}

Animals were killed under pentobarbital anesthesia. The testis and epididymis were dissected out en bloc, fixed by immersion in $4 \%$ paraformaldehyde in $0.1 \mathrm{M}$ phosphate buffer $(\mathrm{pH} 7.4)$ (PFA) or Bouin's solution overnight, dehydrated in a graded ethanol series and embedded in paraffin. Serial $5 \mu \mathrm{m}$-thick sections were made using a microtome with intervals of 10,20 and $50 \mu \mathrm{m}$ for the testes of P0, P21 and adult mice respectively, and then mounted on glass slides.

\section{Immunohistochemistry (IHC)}

Sections of PFA-fixed, paraffin-embedded testes, after deparaffinization and rehydration, were immersed in $20 \mathrm{mM}$ Tris- $\mathrm{HCl}$ buffer $(\mathrm{pH} 9.0)$ and heated at $95^{\circ} \mathrm{C}$ for 15 min using the autoclave apparatus to retrieve antigens (Nakata et al. 2015a). After cooling to room temperature, sections were washed in PBS and treated with $1 \%$ skim milk/PBS at room temperature for $1 \mathrm{~h}$ to prevent non-specific antibody binding. They were then incubated with a rabbit polyclonal anti-laminin antibody (L9393; Sigma-Aldrich) at a concentration of $7.5 \mu \mathrm{g} /$ $\mathrm{mL}$ in PBS at $4^{\circ} \mathrm{C}$ overnight. After washing in PBS, sections were incubated with an Alexa Fluor 594-conjugated donkey anti-rabbit lgG antibody (Molecular Probes) at a 1:400 dilution at room temperature for $1 \mathrm{~h}$. Sections of P21 testes were further incubated with the Alexa Fluor 488 conjugate of peanut agglutinin (PNA) (Molecular Probes), a marker of the acrosome
(Nakata et al. 2015a, 2017), at a 1:400 dilution at room temperature for $30 \mathrm{~min}$. All sections were counterstained in the nuclei with DAPI (4'6-diamidino-2-phenylindole; Molecular Probes) at $300 \mathrm{nM}$, mounted on glass slides with Fluoromount (Diagnostic BioSystems, Pleasanton, CA, USA) and examined with a fluorescence microscope (BX50/BX-FLA; Olympus, Tokyo, Japan, or and BZ-X710; Keyence, Osaka, Japan).

\section{Periodic acid-Schiff-hematoxylin (PAS-H) staining}

Sections of Bouin-fixed, paraffin-embedded testes from adult mice, after deparaffinization and rehydration, were immersed in $0.5 \%$ periodic acid solution for $15 \mathrm{~min}$ and rinsed in running tap water and several changes of distilled water. They were immersed in Schiff's reagent (Merck) for $60 \mathrm{~min}$ and then treated with sulfur water $(12 \mathrm{~mL}$ of sodium bisulfite solution $(10 \%), 10 \mathrm{~mL}$ of hydrochloric acid $(1 \mathrm{~N})$ and $200 \mathrm{~mL}$ distilled water) three times for 3 min each. After rinsing in running tap water and several changes of distilled water, sections were stained with hematoxylin and mounted on glass slides with Multi Mount 220 (Matsunami Glass, Osaka, Japan). All sections were digitized using a whole slide scanner (Nanozoomer 2.0-HT; Hamamatsu Photonics, Hamamatsu, Japan) with the 20 -fold objective lens, and the resulting digital images of the sections were visualized with viewer software (NDP.view2; Hamamatsu Photonics).

\section{Reconstruction processing}

The 3D reconstruction of the seminiferous tubules from serial sections was performed as previously described (Nakata et al. $2015 b)$ with a slight modification. Briefly, digital images of sections of P0 and P21 testes stained with fluorescent IHC for laminin, and of adult testes stained with PAS-H, were converted into grayscale in the JPEG format with Photoshop CS6 software (Adobe Systems) at a resolution of 346.74, 758, and $2724 \mathrm{~nm} /$ pixel, respectively. Using Amira 6.2 .0 software (Visage Imaging $\mathrm{GmbH}$, Berlin, Germany), the inside of the basement membrane of the selected tubule was filled with a particular color using threshold processing and traced from section to section. This procedure was repeatedly applied to all seminiferous tubules with different colors and they were then $3 \mathrm{D}$ reconstructed. The core lines of all reconstructed seminiferous tubules were also drawn with the same software. The position of the rete testis was defined as the mean coordinate of the connections of all reconstructed seminiferous tubules with the rete testis and shown with a gray sphere. Regarding P21 testes, acrosomes were stained in each section with PNA lectin histochemistry (Nakata et al. 2015a) and then reconstructed automatically over the entire testis and superimposed on the 3 D-reconstructed seminiferous tubules in an aligned axis. The core lines of these tubules were divided into portions with the presence and absence of acrosomes. With respect to adult testis, the stages of seminiferous tubules in each section were assessed by histological observations of cell associations and core lines were divided into portions with different stages. The process of $3 \mathrm{D}$ reconstruction of seminiferous tubules is summarized in a flow chart (Supplementary Fig. 1, see section on supplementary data given at the end of this article). 


\section{Statistical analysis}

Statistical differences among multiple values were evaluated with a 1-factor or 2-factor analysis of variance (ANOVA) followed by Bonferroni's post hoc test and differences with a $P$ value less than 0.05 were considered to be significant.

\section{Results}

\section{D structure of all seminiferous tubules in PO mice}

In each of the serial sections of P0 testes, the basement membrane and nuclei were labeled with fluorescence $\mathrm{IHC}$ for laminin and DAPI staining respectively (Fig. 1A and $\mathrm{B})$, and a combined fluorescence image was converted to a grayscale image with reversed gradation (Fig. 1C). The laminin stain was used for the segmentation of the basement membrane, while the nuclear stain contributed to distinguishing seminiferous tubules from blood vessels and other structures and also identifying a continuous seminiferous tubule accurately in serial sections. The inside of the basement membrane of the selected seminiferous tubule was filled with a particular color using threshold processing, traced from section to section along the entire length of the tubule and then 3D-reconstructed automatically. All seminiferous tubules in 3 testes from 3 different $\mathrm{P} 0$ mice (\#1, \#2, and \#3) were reconstructed and the core lines were drawn. The results of \#3 testis with 13 seminiferous tubules were shown in Fig. 1D and E. A single seminiferous tubule was defined as a structure connected to the rete testis with at least one extremity and having a continuous lumen, regardless of the presence of branches or blind ends (Nakata et al. 2015b). The average numbers of seminiferous tubules, terminating points (connections with the rete testis) and branching points per testis in P0 testes were 12.0, 37.0 and 13.3 respectively (Table 1 ). Within all 36 tubules from 3 testes, only one blind end was found. The tubules without branches, with only one branch, and with two or more branches occupied 39, 33 and 28\% of all tubules respectively. The average length of a single seminiferous tubule with the standard deviation was $5.77 \pm 2.94 \mathrm{~mm}$, with the longest tubule being $13.82 \mathrm{~mm}$ and the shortest being $0.59 \mathrm{~mm}$. Except for one tubule with a blind end, all tubules were connected to the rete testis with both extremities. Some branches also terminated in the rete testis, while others connected between two branching points of the respective main tubules.

Regarding the representative P0 testis \#3 in the horizontal (Fig. 2A) and vertical (Fig. 2B) directions, the pathways of most tubules were zigzag in shape, but, as a whole, formed circles along the surface of the testis, with the outer turns of zigzag convolutions being in close association with the tunica albuginea. The amplitude of the zigzag convolution was still shallow at
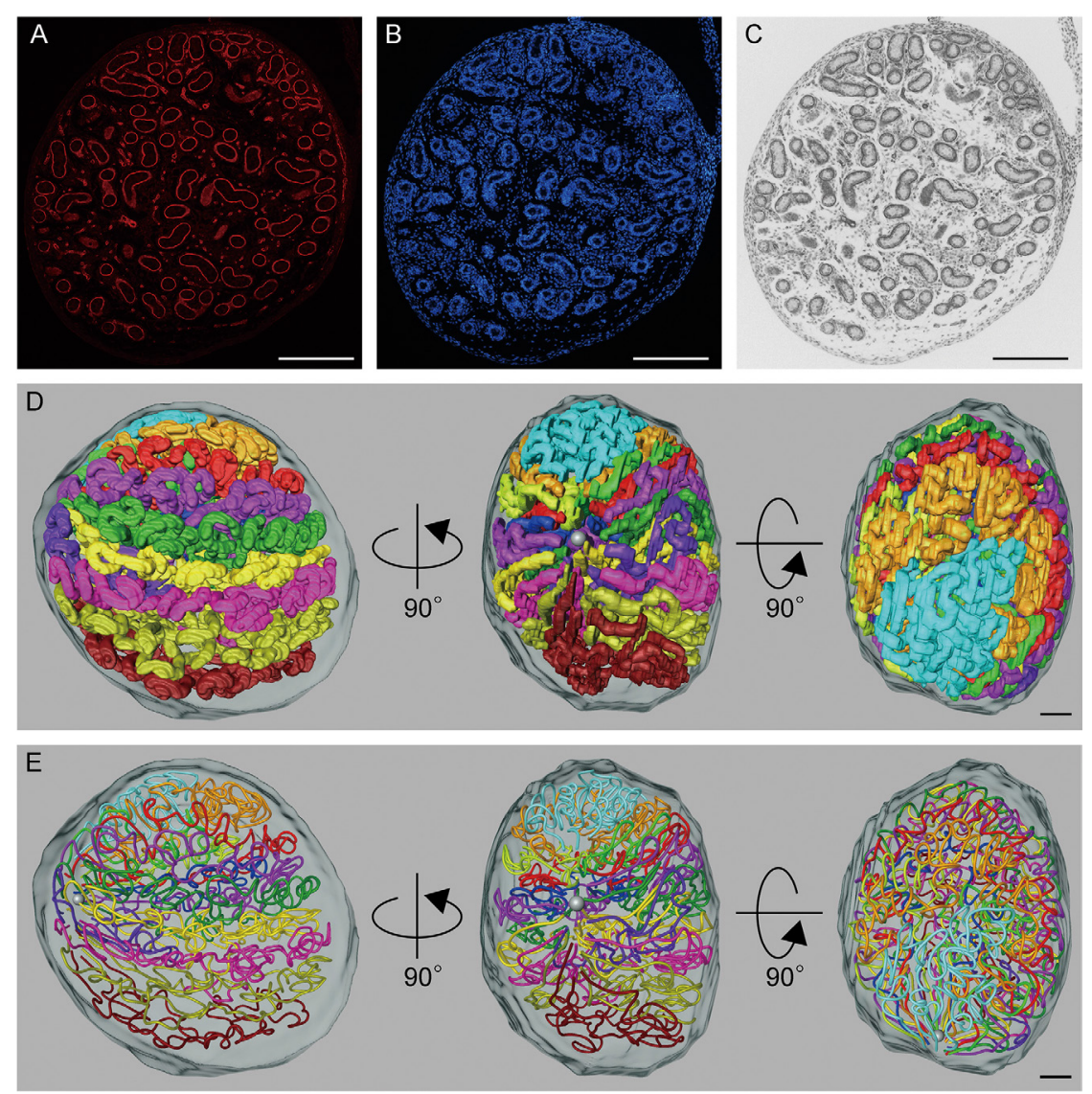

Figure 1 Reconstruction of all seminiferous tubules in P0 mice. Fluorescence immunohistochemistry for laminin (red; A), DAPI staining (blue; B) and a merged image converted into grayscale with reversed gradation $(\mathrm{C})$ are shown for $\# 3$ testis. Reconstructed seminiferous tubules (D) and their core lines (E) are shown with different colors. The position of the rete testis is shown as a gray sphere (D and E). All scales, $100 \mu \mathrm{m}$. 
Table 1 Summary of reconstructed seminiferous tubules in mouse testes. Data are presented as number or number (\%).

\begin{tabular}{|c|c|c|c|c|c|c|c|c|c|}
\hline \multirow[b]{2}{*}{ Testis } & \multirow[b]{2}{*}{ Age } & \multirow[b]{2}{*}{ Total tubules } & \multirow[b]{2}{*}{$\begin{array}{c}\text { Terminating } \\
\text { points* }\end{array}$} & \multirow[b]{2}{*}{$\begin{array}{c}\text { Branching } \\
\text { points }\end{array}$} & \multirow[b]{2}{*}{ Blind ends } & \multicolumn{3}{|c|}{ Tubules with } & \multirow[b]{2}{*}{$\begin{array}{l}\text { Average tubule } \\
\text { length** }(\mathrm{mm})\end{array}$} \\
\hline & & & & & & $\begin{array}{c}\text { No branches } \\
(\%)\end{array}$ & $\begin{array}{c}\text { One branch } \\
(\%)\end{array}$ & $\begin{array}{l}\text { Two or more } \\
\text { branches (\%) }\end{array}$ & \\
\hline$\# 1$ & 0 & 11 & 32 & 10 & - & $5(45)$ & $4(36)$ & $2(18)$ & $5.16 \pm 2.81$ \\
\hline$\# 2$ & 0 & 12 & 41 & 18 & 1 & $3(25)$ & $4(33)$ & $5(42)$ & $6.45 \pm 3.65$ \\
\hline$\# 3$ & 0 & 13 & 38 & 12 & - & $6(46)$ & $4(31)$ & $3(23)$ & $5.67 \pm 2.00$ \\
\hline$\# 4$ & 21 & 11 & 31 & 9 & - & $5(45)$ & $5(45)$ & $1(9)$ & $63.6 \pm 31.2$ \\
\hline$\# 5$ & 21 & 12 & 36 & 12 & - & $5(42)$ & $5(42)$ & $2(17)$ & $57.8 \pm 29.5$ \\
\hline$\# 6$ & 21 & 13 & 43 & 21 & - & $5(38)$ & $6(46)$ & $2(15)$ & $63.0 \pm 62.8$ \\
\hline$\# 7$ & 90 & 9 & 38 & 20 & - & $2(22)$ & $4(44)$ & $3(33)$ & $198 \pm 101$ \\
\hline$\# 8$ & 90 & 11 & 39 & 19 & - & $-(0)$ & $8(73)$ & $3(27)$ & $118 \pm 35$ \\
\hline$\# 9$ & 90 & 16 & 49 & 19 & - & $7(44)$ & $4(25)$ & $5(31)$ & $109 \pm 66$ \\
\hline
\end{tabular}

${ }^{*}$ Connections with the rete testis; ${ }^{* *}$ Mean \pm S.D.

P0 in the horizontal and vertical directions, resulting in broad central areas of the testis being unoccupied by tubules in the horizontal view and the simple piling up of disc-shaped tubule networks in the cranial to caudal direction in the vertical view. In T7, T9, T11 and T12 tubules, minor portions that branched from the major portions had pathways running all the way in the center of the testis far from the tunica albuginea and terminating in the rete testis.

\section{D structure of all seminiferous tubules and acrosomes in P21 mice}

All seminiferous tubules in 3 testes from 3 different P21 mice (\#4, \#5 and \#6) were reconstructed and the core lines were drawn, using the same method described previously. The results of $\# 4$ testis with 11 seminiferous tubules were shown in Fig. 3. The average numbers of seminiferous tubules, terminating points and branching points per testis were $12.0,36.7$ and 14.0 respectively (Table 1). No blind end was found within all 36 tubules from 3 testes. The tubules without branches, with only one branch, and with two or more branches occupied 41,44 and $14 \%$ of all tubules respectively. None of the values for the numbers of seminiferous tubules, terminating points and branching points, or frequencies of tubule types were significantly different from those in P0. The average length of a single seminiferous tubule was $61.4 \pm 44.9 \mathrm{~mm}$, with the longest tubule being $246.0 \mathrm{~mm}$ and the shortest being $4.1 \mathrm{~mm}$. This value was significantly larger than that in P0 $(P<0.01)$. The zigzag convolutions of tubules became more constant in direction, and their amplitude became markedly larger than that in P0 in both the horizontal and vertical

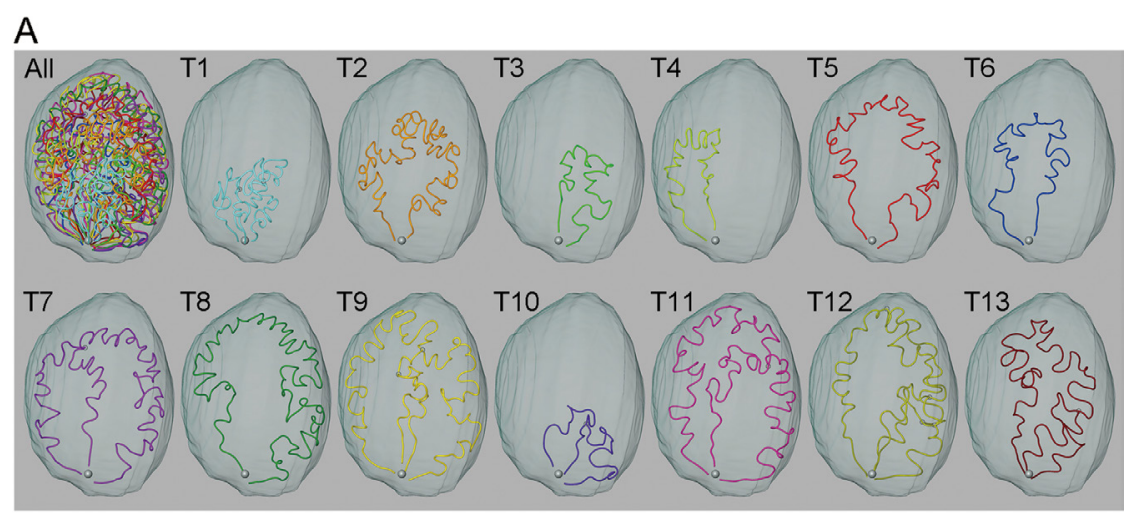

B

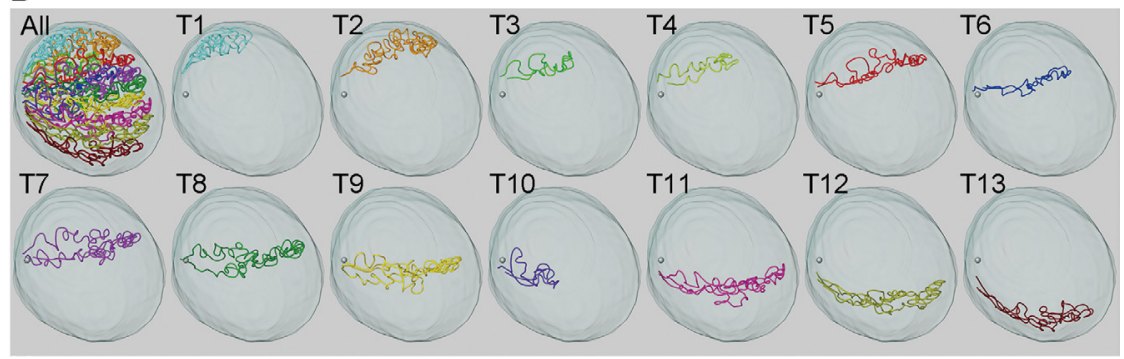

Figure 2 Core lines of all reconstructed seminiferous tubules in P0 mice. The core lines of individual seminiferous tubules named T1 to T13 in the order of their connection with the rete testis (gray sphere) from the top toward the bottom in $\# 3$ testis are shown in horizontal (A) and vertical (B) views. 


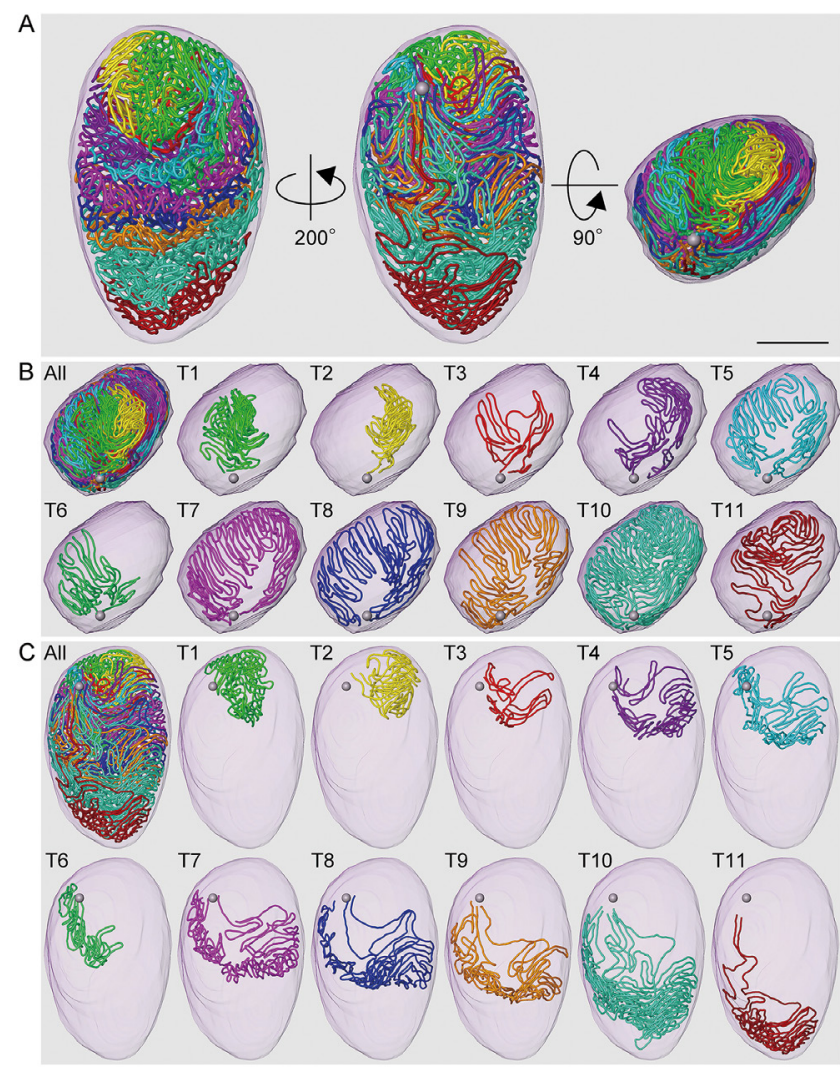

Figure 3 Core lines of all reconstructed seminiferous tubules in P21 mice. The core lines of all reconstructed seminiferous tubules (A) and of individual tubules named T1 to T11 in the order of their connection with the rete testis (gray sphere) from the top toward the bottom in \#4 testis are shown in horizontal (B) and vertical (C) views. Scale, $1 \mathrm{~mm}(\mathrm{~A})$.

directions. The cranial turns of convolutions were in contact with the tunica albuginea, whereas the caudal turns occupied the central areas of the testis. As a whole, all seminiferous tubules formed funnel-shaped networks that tapered toward the caudal direction, and caudally located networks surrounded the preceding cranially located networks from the bottom and outside with the appearance of stacked paper cups.

Since the round spermatid initially occurs at P20 (Drumond et al. 2011), the acrosome, a special structure of the spermatid, is expected to appear for the first time at P21. When PNA lectin histochemistry was applied to the sections of representative P21 testis \#4, acrosomes were only found in a small subpopulation of seminiferous tubules (Fig. 4A and B), suggesting that it represents the tubule portions at which spermatogenesis initially occurred in the early postnatal period and had advanced the most by P21. In order to visualize these portions in $3 \mathrm{D}$ images, acrosomes in serial sections of P21 testes were reconstructed automatically and superimposed on reconstructed seminiferous tubules by aligning the axis. Regarding the representative results of \#6 testis, acrosomes were not distributed uniformly within the
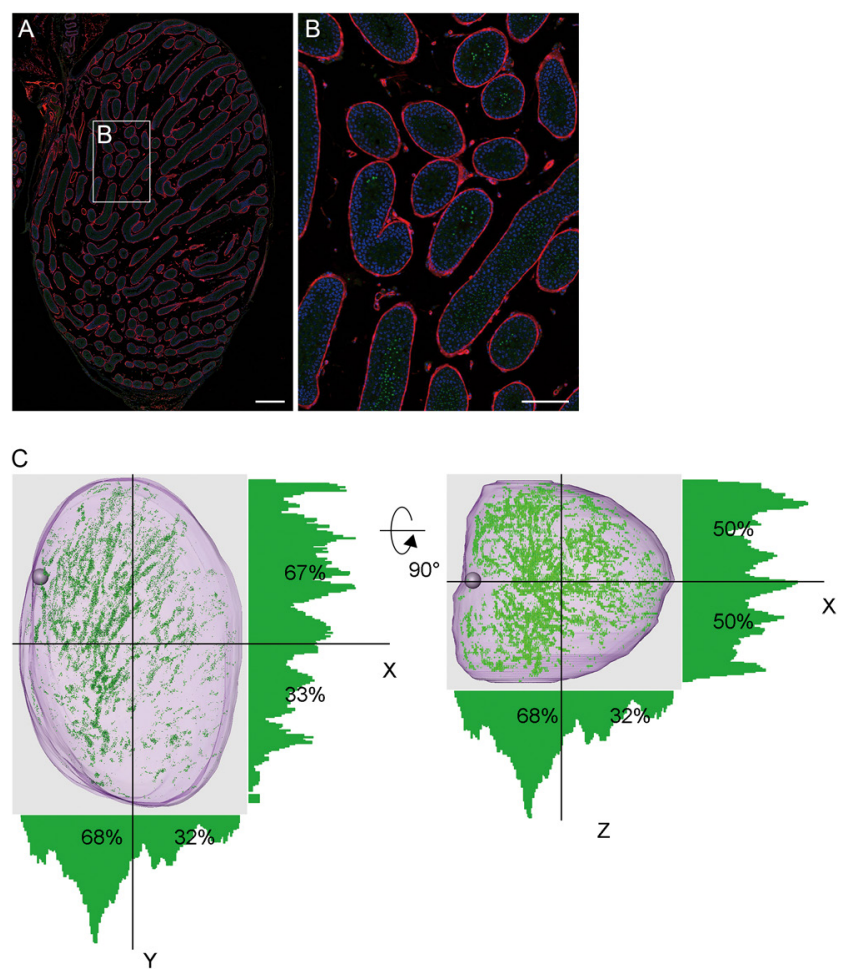

Figure 4 Reconstruction of acrosomes in P21 mice. Combination of fluorescence immunohistochemistry for laminin (red), lectin histochemistry with fluorescent peanut agglutinin (green), and DAPI staining (blue) are shown (A and B). Reconstructed acrosomes and the position of the rete testis in $\# 6$ testis are shown as green dots and a gray sphere, respectively $(\mathrm{C})$. The relative volumes of acrosomes in $20-\mu \mathrm{m}$-thick slices of a testis parallel to the 3 planes formed by the $X$, $Y$ and $Z$ axes against the volumes of respective slices (i.e., densities of acrosomes) are plotted in bar graphs. The numerals shown in the graphs represent the volumes of acrosomes in the areas of the testis divided by the 3 planes against the total volume of acrosomes in the testis (\%). Scales, $300 \mu \mathrm{m}(\mathrm{A}), 100 \mu \mathrm{m}(\mathrm{B})$.

testis (Fig. 4C). Therefore, a quantitative analysis of the 3D distribution of acrosomes was performed in the testis with the rete testis situated at the upper left corner, as in Fig. 4C. Along the planes formed by the $x-y-z$ axes, the summed volumes of acrosomes contained in serial $20 \mu \mathrm{m}$-thick slices of the testis against the total volume of the corresponding slices (i.e., densities of acrosomes) were shown in bars. The density of acrosomes, which represents the number of sites at which spermatids initially occurred in the testis, was 2.0-fold larger in the upper than in the lower regions of the testis divided by the $X-Z$ plane, and 2.1 -fold larger in the left (i.e., proximal to the rete testis) than that in the right (distal) regions divided by the $Y-Z$ plane, suggesting that it is larger in the tubule portions closer to the rete testis. In contrast, no significant difference was observed in the density of acrosomes between the regions divided by the $X-Y$ plane. When the testis was divided into 4 regions, the relative density of acrosomes in the upper proximal, upper distal, lower proximal and lower distal regions 
was calculated to be 4.3:2.0:2.1:1. Similar results were obtained in two other testes from P21 mice (\#4 and \#5). The portions of seminiferous tubules containing acrosomes were superimposed on the core lines of all 11 tubules in \#4 testis (Fig. 5A and B). The portions containing acrosomes occupied $20 \%$ of the length of each tubule on average in upper (cranial) tubules T1 to T8, but only 7\% in lower (caudal) end tubules T9 to T11. Within individual seminiferous tubules, the portions containing acrosomes were distributed more abundantly on both sides of tubules closer to the rete testis than in the middle portions of tubules far from the rete testis.

\section{D structure of all seminiferous tubules and waves in adult mice}

In the case of P90 (adult) testis, possibly because it was large and had thick tunica albuginea, the immersion fixation in PFA adopted for P0 and P21 testes produced poorly fixed specimens, in which seminiferous tubules were distorted in shape and their outlines were obscure. Furthermore, the results of $\mathrm{IHC}$ and lectin histochemistry were uneven from portion to portion of the sections, making it difficult to segment seminiferous tubules in serial sections and reconstruct them accurately. Therefore, we applied PAS-H staining to specimens fixed with Bouin's solution, which produces better fixation, but slightly inactivates reactivity for $\mathrm{IHC}$ or lectin histochemistry. In serial sections of adult testes stained with PAS- $\mathrm{H}$, the basement membrane and acrosomes were labeled red with PAS, while the nuclei were labeled blue with hematoxylin (Fig. 6A and B). The basement membrane was segmented by converting PAS-H images, after increasing the relative intensity of the red channel to the blue channel, to grayscale images (Fig. 6C). Thereafter, the inside of the basal membrane of a particular seminiferous tubule was filled with a particular color, traced from section to section and $3 \mathrm{D}$ reconstructed in the same manner as for the P0 and P21 testes. The nuclear stain with hematoxylin as well as the acrosomal stain with PAS contributed to identifying a continuous seminiferous tubule accurately in serial sections.

All seminiferous tubules in 3 testes from 3 adult mice $(\# 7, \# 8$ and $\# 9)$ were reconstructed and the core lines were drawn. The results of $\# 7$ testis with 9 seminiferous tubules were shown in Figs $6 \mathrm{D}$ and $7 \mathrm{~A}$ and $\mathrm{B}$. The average numbers of seminiferous tubules, terminating points and branching points per testis were 12.0, 42.0 and 19.3 respectively (Table 1 ). No blind end was found within all 36 tubules from 3 testes. The tubules without branches, with only one branch, and with two or more branches occupied 25, 44 and $31 \%$ of all tubules respectively. None of the values for the numbers of seminiferous tubules, terminating points and branching points, or frequencies of tubule types were significantly different from those in P0 and P21. The average length of a single seminiferous tubule was $134 \pm 79 \mathrm{~mm}$, with the longest tubule being $386 \mathrm{~mm}$ and the shortest being $6 \mathrm{~mm}$. This value was significantly larger than that in PO and P21 $(P<0.01)$. The amplitude of the zigzag pathway of tubules became larger and the 'stacked cups' pattern
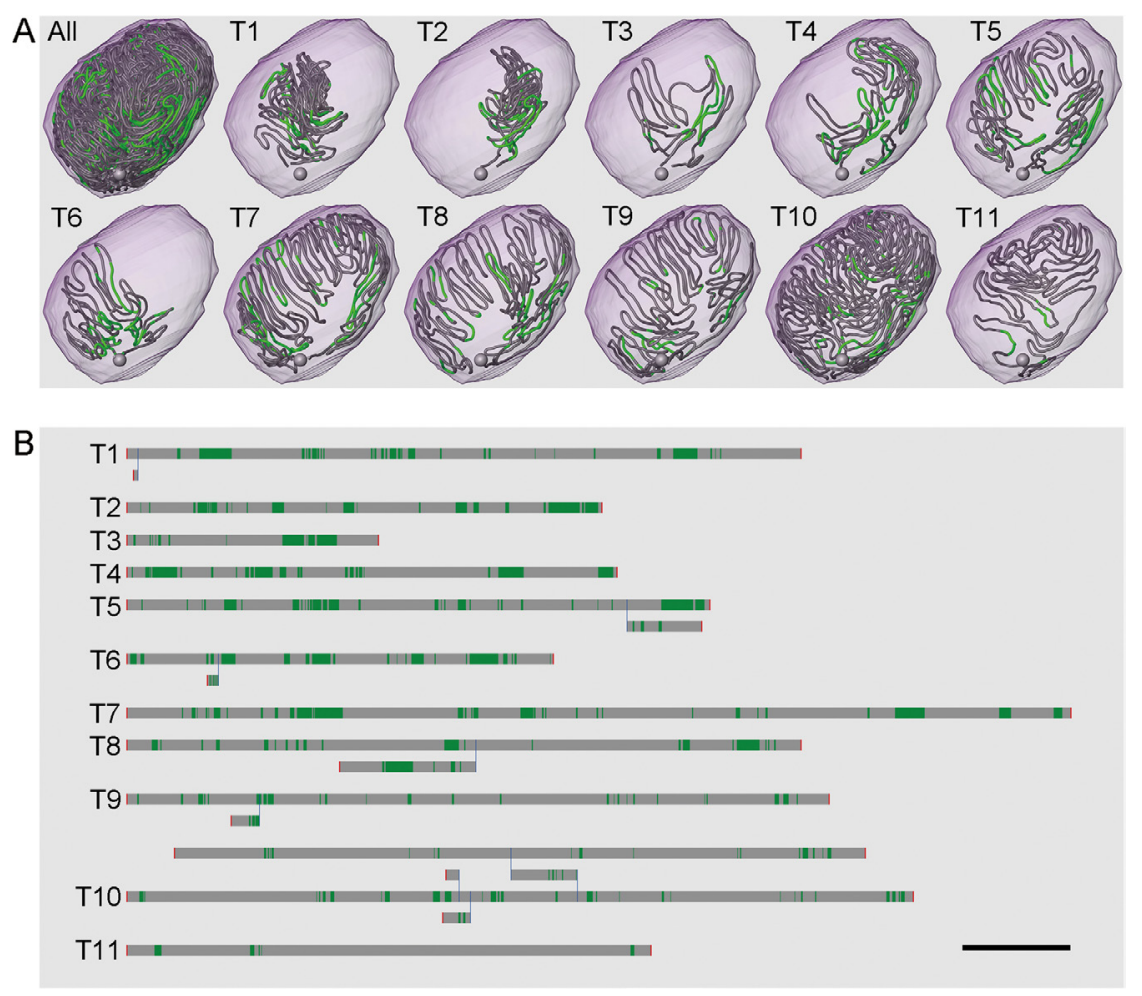

Figure 5 Distribution of portions of seminiferous tubules containing acrosomes in P21 mice. The core lines of all reconstructed seminiferous tubules in \#4 testis are shown in a horizontal view with the portions of tubules containing acrosomes marked in green and the position of the rete testis shown with a gray sphere (A). The scheme of individual seminiferous tubules is shown in (B). The portions of tubules containing acrosomes are marked in green, and the branching points are shown with vertical bars. All free extremities of the tubules including the branches on both sides are connected to the rete testis. Scale, $10 \mathrm{~mm}(\mathrm{~B})$. 

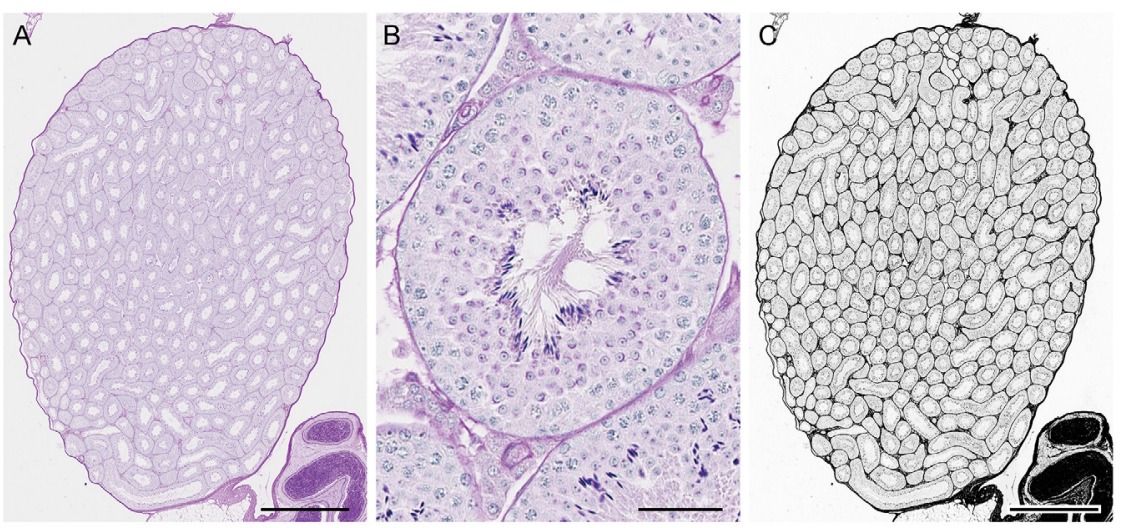

D
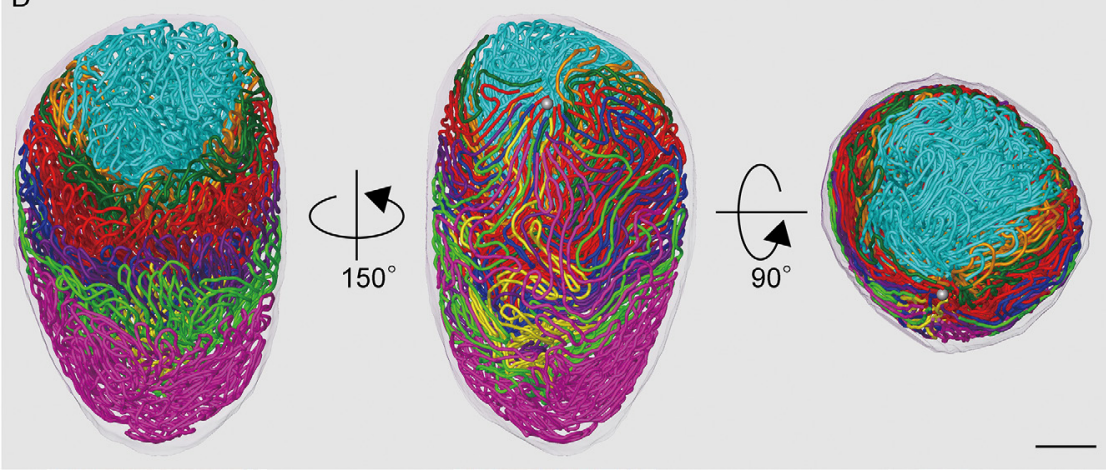

Figure 6 Reconstruction of all seminiferous tubules in P90 (adult) mice. PAS-H-stained sections with low (A) and high magnifications (B) and a grayscale image of A with an emphasized PAS-positive basement membrane (C) are shown. The core lines of all reconstructed seminiferous tubules of $\# 7$ testis with the position of the rete testis (gray sphere) are shown in (D). Scales, $1 \mathrm{~mm}(\mathrm{~A}, \mathrm{C}, \mathrm{D})$, $50 \mu \mathrm{m}(\mathrm{B})$. of the tubule networks tapering toward the caudal direction became more prominent than those in P21.

In order to analyze waves in the seminiferous epithelium, the stages of all seminiferous tubules in each section were assessed for 80 sections from a representative adult testis $\# 7$. Since PAS-H stained not only the basement membrane, but also the acrosome, it was possible to evaluate all 12 stages based on the cell association and shapes of acrosomes (Oakberg 1956). The stages of the seminiferous epithelium generally aligned in a descending manner to the direction at which the wave proceeded, i.e., a lower stage number is located distal to the preceding higher stage number within a seminiferous tubule; however, this basic pattern of the arrangement of stages is often interrupted by local irregularities in the order of stage numbers called 'modulations' (Perey et al. 1961). In addition, individual stages were too short in length to visualize and analyze in 3D-reconstructed images. Therefore, in order to avoid complexity, we divided the seminiferous epithelium into 3 groups, i.e., stages I-VI, which had two generations of spermatids, but no elongated spermatids lining the lumen of the seminiferous epithelium, stages VII-VIII, which had two generations of spermatids and elongated spermatids lining the lumen and stages IXXII, which had only one generation of spermatids.

The core lines of all seminiferous tubules in $\# 7$ testis were divided into these 3 groups with different colors
(Fig. 8A and B and Table 2). Seventy-six complete waves were detected, and the average length of a single wave was $16.9 \pm 7.8 \mathrm{~mm}$, with the longest wave being $34.2 \mathrm{~mm}$ and the shortest being $2.5 \mathrm{~mm}$. Since most tubules had 2 or more extremities connected to the rete testis, there was one or more point(s) at which two or more lines of stages descending from the rete testis met. This point, at which the direction of the wave reverses, was called the 'site of reversal' by previous investigators (Perey et al. 1961). We found 19 sites of reversal in the 9 seminiferous tubules in \#7 testis. In the T6 and T7 tubules, which had no branches, and in T3, T5 and T8, which had only short branches, there was only one site of reversal per tubule. In T1 and T4, which had complex pathways with many branches, there were many sites of reversal per tubule. T9 had many branches, but only 2 sites of reversal, whereas T2 had only a short branch, but 3 sites of reversal. These results revealed that the site of reversal never occurs in the branching point, at which the wave always proceeds continuously in the same direction in the two following branches. Furthermore, when a single tubule was traced from its one terminus in the rete testis to the other via any of the branches, the number of sites of reversal encountered was always an odd number (1, 3 or 5$)$, as theoretically expected. These sites of reversal, even in the case of one site per tubule, were not necessarily located at equal distances from both extremities of the tubules connected to the rete testis. 


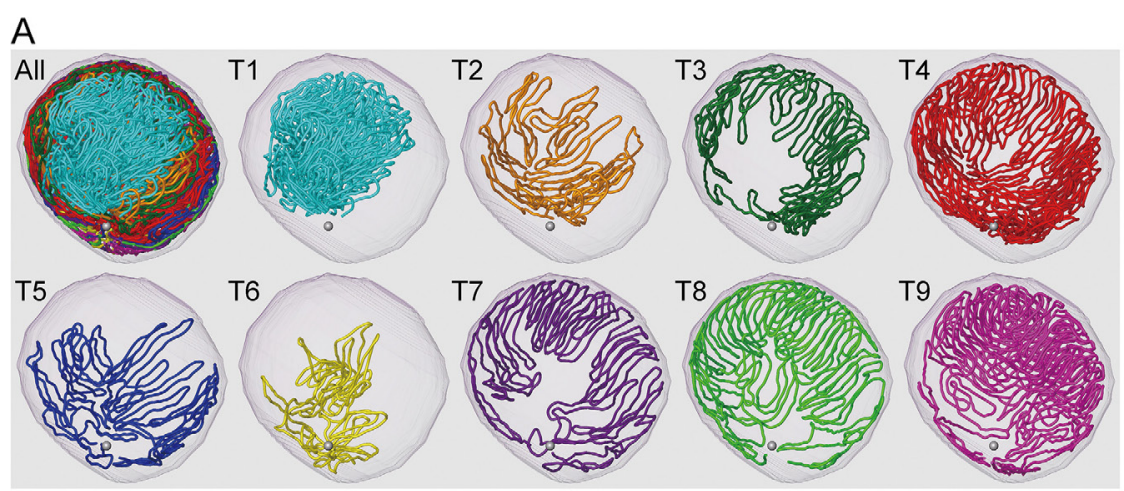

B

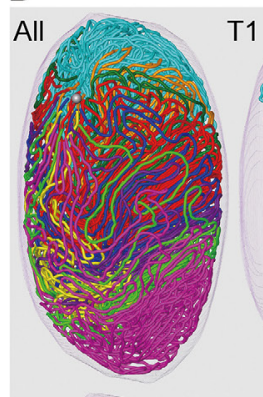

T5 T6

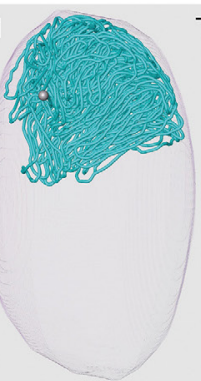

T2

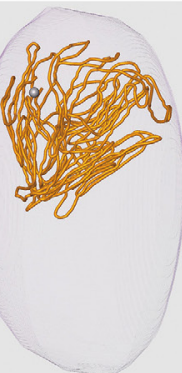

T3

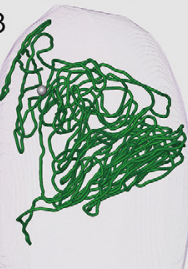

$\mathrm{T} 4$

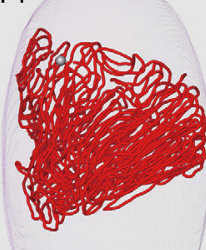

\begin{tabular}{ll}
$\mathrm{T} 7$ \\
\hline
\end{tabular}
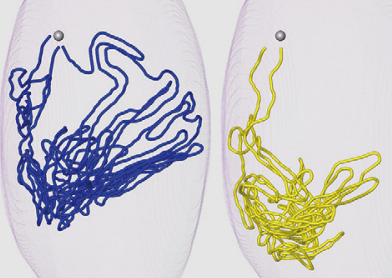

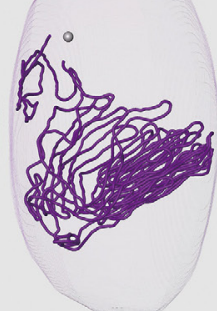

T8

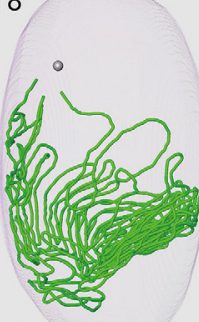

Figure 7 Core lines of all reconstructed seminiferous tubules in P90 (adult) mice. The core lines of individual seminiferous tubules named T1 to T9 in the order of their connection with the rete testis (gray sphere) from the top toward the bottom in \#7 testis are shown in horizontal (A) and vertical (B) views.

\section{Discussion}

Our procedure for the 3D reconstruction of seminiferous tubules is composed of 3 steps: (1) the segmentation of seminiferous tubules by marking the outlines of all tubules in digital images of serial testis sections; (2) the tracing of individual seminiferous tubules along their entire length by labeling a continuous tubule from section to section with a particular color and repeating it for all seminiferous tubules with different colors and (3) the reconstruction of individual labeled seminiferous tubules from serial sections with high-performance 3D reconstruction software. In our previous study (Nakata et al. 2015b), we conducted step (1) by marking the outlines of seminiferous tubules manually from section to section on the monitor. In the present study, we labeled the basement membrane of all seminiferous tubules in serial sections at once with fluorescent IHC or PAS-H staining for the purpose of step (1). Although subsequent step (2), which involves identifying continuous individual seminiferous tubules and filling the inside of tubules in color using threshold processing from section to section on the monitor, was unchanged from the previous study, and the improvement of step (1) greatly reduced the labor and time required for the whole procedure.

The combination of whole mount fluorescence labeling and confocal microscopy has been used for $3 \mathrm{D}$ analysis of the cellular composition in individual seminiferous tubules (Ehmcke et al. 2005, Ikami et al. 2015) as well as the structure of embryonic sex cords (Combes et al. 2009, Nel-Themaat et al. 2009). However, confocal microscopy, using the optical sectioning, can obtain images within a limited depth from the tissue surface and is unsuitable for thick specimens such as whole adult testis. The present method has the advantage in using ordinary serial sections obtained from formalinfixed, paraffin-embedded specimens that are common in human pathology.

In the present study, all seminiferous tubules in 3 testes each at 0,21 and 90 days (adult) postpartum were reconstructed and their 3D structures were analyzed. Detailed quantitative information was obtained, including the numbers of seminiferous tubules, terminating points (connections with the rete testis), and branching points per testis, as well as the frequency of 


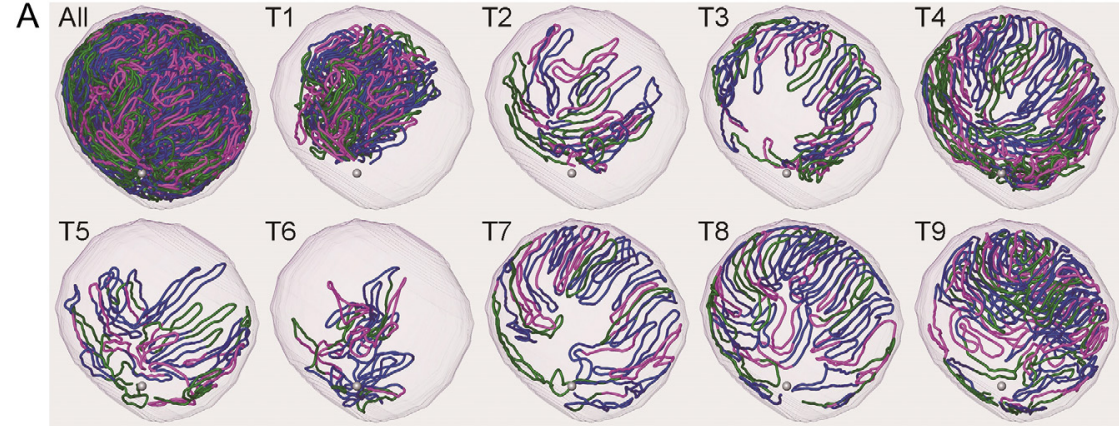

B

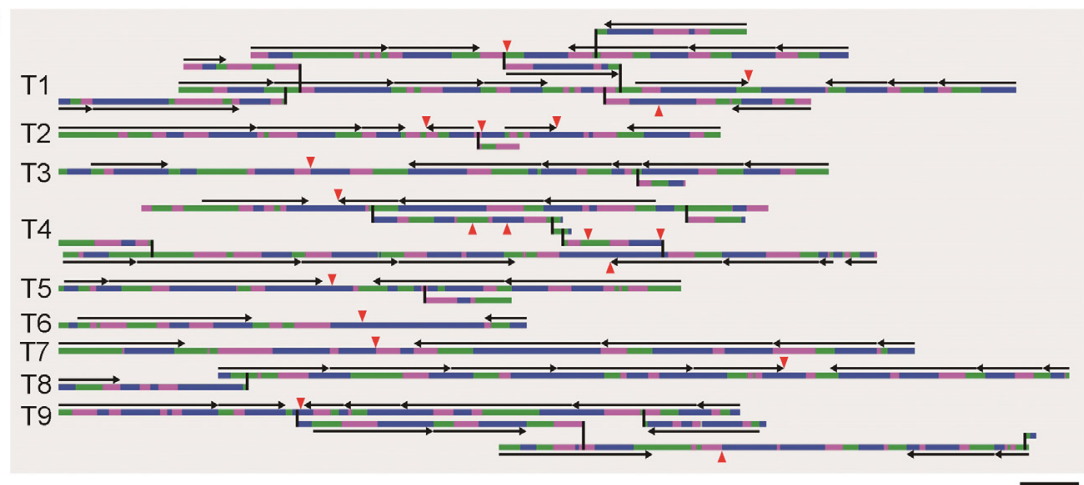

Figure 8 Distribution of spermatogenic stages and waves in seminiferous tubules in P90 (adult) mice. The core lines of all reconstructed seminiferous tubules in $\# 7$ testis are shown in a horizontal view with the portions in 3 different stage groups being marked in different colors and the position of the rete testis shown with a gray sphere (A). The 3 different stage groups are as follows: stages I-VI (blue), stages VII-VIII (pink) and stages IX-XII (green). The scheme of individual seminiferous tubules is shown in (B). The portions of tubules in different stage groups are marked in the colors shown above, the branching points shown with vertical bars, the sites of reversal shown with red triangles and the ranges and directions of complete waves shown with arrows. All free extremities of the tubules including the branches on both sides are connected to the rete testis. Scale, $10 \mathrm{~mm}$ (B). tubules with different numbers of branches. Although marked variations in these values existed among individual testes, the averages of these values were not significantly different among the ages tested, suggesting that the basic regular arrangement of seminiferous tubules is established by P0 and retained until adulthood. On the other hand, the average length of a seminiferous tubule showed a marked increase postnatally, ranging approximately 25-fold between P0 and P90. The results of the present study clearly demonstrated that as seminiferous tubules become longer during postnatal development, they become more tightly packed in the testis by increasing the amplitude of their zigzag paths and occupying more space in the center of the testis. Furthermore, the characteristic 'stacked cups' pattern of seminiferous tubules, in which caudally located funnel-shaped tubule networks surround preceding cranially located networks from the bottom and outside (Clermont \& Huckins 1961, Nakata et al. 2015b), was not yet apparent at P0, but developed to some extent by P21 and then became apparent in adults. The biological meaning of multiple funnel-shaped tubule networks stacked like paper cups, instead of the single winding tubule, may reside in the efficiency of packing longer seminiferous tubules and transporting larger numbers of sperm to the rete testis.

Clermont and Huckins (1961) analyzed 20 seminiferous tubules in an adult rat testis and reported the presence of 'outer' and 'inner' types, with only the former being in contact with the tunica albuginea. Our present results, together with our previous findings (Nakata et al. 2015b), demonstrated that, as far as a single

Table 2 Summary of the stage of and waves in seminiferous tubules in an adult mouse testis.

\begin{tabular}{|c|c|c|c|c|c|c|}
\hline \multirow[b]{3}{*}{ Tubule } & \multicolumn{4}{|c|}{ Total length $(\mathrm{mm})$ of } & \multirow{2}{*}{\multicolumn{2}{|c|}{ Complete wave ${ }^{* *}$}} \\
\hline & & & ge groups $(\%)$ & & & \\
\hline & Whole tubule & $\mathbf{I}-\mathrm{VI}$ & VII-VIII & IX-XII & Number & Average length $(\mathrm{mm})$ \\
\hline $\mathrm{T} 1 *$ & 386.5 & $172.1(44.5)$ & $109.8(28.4)$ & $104.6(27.1)$ & 19 & $15.8 \pm 5.5^{+}$ \\
\hline T2 & 120.7 & $47.9(39.7)$ & $37.5(31.0)$ & 35.3 (29.3) & 6 & $15.5 \pm 9.3$ \\
\hline T3 & 140.9 & $68.2(48.4)$ & $26.0(18.4)$ & $46.7(33.2)$ & 6 & $14.3 \pm 5.4$ \\
\hline T4 & 328.5 & $129.5(39.4)$ & $89.6(27.3)$ & $109.5(33.3)$ & 12 & $16.2 \pm 7.1$ \\
\hline T5 & 122.2 & $58.3(47.8)$ & $27.8(22.8)$ & $36.0(29.5)$ & 4 & $24.4 \pm 10.9$ \\
\hline T6 & 80.6 & $40.9(50.7)$ & $21.9(27.2)$ & $17.8(22.0)$ & 2 & $18.7 \pm 11.4$ \\
\hline $\mathrm{T7}$ & 147.2 & $79.3(53.9)$ & $34.6(23.5)$ & $33.2(22.6)$ & 5 & $21.6 \pm 9.1$ \\
\hline T8 & 178.6 & $97.4(54.5)$ & $37.6(21.0)$ & $43.7(24.5)$ & 9 & $16.7 \pm 6.2$ \\
\hline T9 & 279.5 & $134.2(48.0)$ & $73.2(26.2)$ & $72.1(25.8)$ & 13 & $16.7 \pm 7.7$ \\
\hline
\end{tabular}

The results of $\# 7$ testis. *T1 to T9 are assigned to tubules in the order of their connection to the rete testis from the top to the bottom; ${ }^{* *}$ Defined as a series of adjacent stages that include all 12 types; ${ }^{\dagger}$ Mean \pm S.D. 
seminiferous tubule is defined as a structure connected to the rete testis at least with one extremity and having a continuous lumen, regardless of the presence of branches or blind ends, all seminiferous tubules from P0 to adult mouse testes were in contact with the tunica albuginea, at least in some portions of their zigzag paths, along the surface of the testis; therefore, we were unable to find the inner type of tubules. However, in the P0 testis, the amplitude of the zigzag convolution was still shallow and most portions of the tubules were located close to the surface of the testis, leaving the large central areas of the testis unoccupied by seminiferous tubules. However, some portions of the tubules, mainly the branches that terminated in the rete testis, were located exclusively in the central areas of the testis. These portions may be regarded as the 'inner' portions of seminiferous tubules, which may represent the counterpart of inner type tubules in rats. In P21 and adult testes, with increases in the amplitude of zigzag convolutions in the seminiferous tubules, the central areas of the testis became occupied with convolutions, and the 'inner' portions of tubules became less apparent, but were still present. The biological meaning of the presence of the two types of seminiferous tubules in the rat and their absence in the mouse is unknown, but may be related to differences in the sizes of testes. Seminiferous tubules in the postnatal testis are derived from embryonic sex cords and Clermont and Huckins (1961) reported that sex cords in rat embryos have inner and outer types. In mouse embryos, there are contradicting findings on the presence (Combes et al. 2009) and absence (NelThemaat et al. 2009) of the two types of sex cords. If inner type sex cords exist in mouse embryos, they may fuse with the outer cords to form branches of the latter by the time of birth, and these branches may become the main constituent of the inner portions of seminiferous tubules in mice. Further studies are required to clarify this issue.

Spermatogenesis begins a few days postpartum when gonocytes divide into undifferentiated spermatogonia (stem cells) and differentiating spermatogonia (Drumond et al. 2011). The process for the first differentiating spermatogonia to give rise to mature sperm is called the first round of spermatogenesis, which is approximately 2.5 days shorter in duration than the established ordinary round of spermatogenesis in adults (Kluin \& de Rooji 1981, Drumond et al. 2011). The onset of spermatogenesis occurs in patches along the length of seminiferous tubules and is dependent on retinoic acid (Ikami et al. 2015, Agrimson et al. 2016). Thereafter, the first round of spermatogenesis advances and is completed with the appearance of mature spermatids at the starting point around 37 days postpartum (Drumond et al. 2011). In the present study, we analyzed the 3D distribution of acrosomes in the P21 mouse testis and found that they were present in patches that were distributed more preferentially in the upper (cranial) than in the lower (caudal) seminiferous tubules in the testis and in portions proximal rather than distal to the rete testis within seminiferous tubules. Since the acrosome is known to become prominent in step 2 spermatids (Russell et al. 1990), the portions positive for acrosomes represent the point at which spermatogenesis has reached the production of round spermatids earliest in the testis. Assuming that the speed of the progression of spermatogenesis does not differ along the length of seminiferous tubules, these portions reasonably correspond to the sites at which the first round of spermatogenesis began in the early postnatal days. The biological meaning and molecular mechanisms responsible for the preferential distribution of the onset of spermatogenesis are unknown, but may be related to efficiency in the transportation of sperm to the rete testis and the local availability of retinoic acid, which is known to initiate spermatogenesis. We also hypothesize that the molecular mechanisms responsible for the postnatal formation of the 'stacked cups' pattern of seminiferous tubule networks are related to the sites of the onset and progression of spermatogenesis.

Regarding spermatogenic waves, Perey and coworkers (1961) conducted a detailed study on rats. They isolated fragments of seminiferous tubules from an adult rat testis, performed fixation and paraffin embedding procedures in a flat glass chamber, and then cut longitudinal sections from the fragments and stained them with PAS-H. Thereafter, they assessed the stages of the seminiferous epithelium and placed the stage numbers on segments along the length of each tubule fragment. While the segments as a whole showed a descending order, i.e., decreasing stage numbers, from the rete testis to the distal portion of tubules, some segments were arranged in an ascending order in the distal direction. This irregularity was called 'modulation', and, thus, the wave was defined in rats as a series of adjacent stage segments that included all 14 possible types, in addition to any segment that may be involved in modulation. Taken together with the analysis of stages in 20 seminiferous tubules using serial sections of an adult rat testis, they reported that the average length of the wave was $26 \mathrm{~mm}$, and the site of reversal, which existed as one per tubule in principle, was located at approximately equal distances from both junctions of a tubule with the rete testis. In the present study, by using the novel 3D reconstruction procedure, we measured, for the first time in an adult mouse testis, 76 complete waves with an average length of $16.9 \mathrm{~mm}$ (ranging between 2.5 and $34.2 \mathrm{~mm}$ ). We also found 19 sites of reversal in a testis, which was larger than the number of seminiferous tubules, 9, because there are more branches in mice than in rats and, accordingly, there are multiple sites of reversal in some tubules. In contrast to rats, the distances from any site of reversal to both junctions of a tubule with the rete testis were not equal. The biological meaning of the presence of 
multiple waves and site of reversal may reside in the continuous production of sperm without time intervals and the efficient transport of sperm to the rete testis, respectively. The molecular mechanisms regulating the spermatogenic wave by factors within or outside of the seminiferous tubules are yet to be elucidated.

The technique used in the present study has enabled a detailed analysis of the structure and function of seminiferous tubules, including the 3D arrangement of tubules and its postnatal changes as well as spermatogenesis in 3D-reconstructed tubules. It will be useful for research in mice and humans under normal and pathological states, such as infertility.

\section{Supplementary data}

This is linked to the online version of the paper at http://dx.doi.org/10.1530/REP-17-0391.

\section{Declaration of interest}

The authors declare that there is no conflict of interest that may be perceived as prejudicing the impartiality of the research reported.

\section{Funding}

This work was supported by MEXT KAKENHI Grant Number JP16K18976.

\section{Acknowledgments}

The authors thank Itsuro Kamimura (Maxnet co., Ltd.) for his advice on using Amira software, Osamu Takahashi (Keyence) and Nobuyuki Yoshioka (Keyence) for the use of BZ-X710, and Dr Toshihisa Hatta (Kanazawa Medical University) and Dr Satoru Honma (Kanazawa Medical University) for the use of Nanozoomer 2.0-HT. They also thank Shuichi Yamazaki for his technical assistance making paraffin sections.

\section{References}

Agrimson KS, Onken J, Mitchell D, Topping TB, Chiarini-Garcia H, Hogarth CA \& Griswold MD 2016 Characterizing the spermatogonial response to retinoic acid during the onset of Spermatogenesis and following synchronization in the neonatal mouse testis. Biology of Reproduction 95 1-15. (doi:10.1095/biolreprod.116.142240)

Ahmed EA \& de Rooij DG 2009 Staging of mouse seminiferous tubule crosssections. Methods in Molecular Biology 558 263-277. (doi:10.1002/ aja.1001080106)

Clermont Y \& Huckins C 1961 Microscopic anatomy of the sex cords and seminiferous tubules in growing and adult male albino rats. American Journal of Anatomy 108 79-97. (doi:10.1002/aja.1001080106)

Combes AN, Lesieur E, Harley VR, Sinclair AH, Little MH, Wilhelm D \& Koopman P 2009 Three-dimensional visualization of testis cord morphogenesis, a novel tubulogenic mechanism in development. Developmental Dynamics 238 1033-1041. (doi:10.1002/dvdy.21925)

Curtis GM 1918 The morphology of the mammalian seminiferous tubule. American Journal of Anatomy 24 339-394. (doi:10.1002/ aja.1000240303)

de Rooij DG \& de Boer P 2003 Specific arrests of spermatogenesis in genetically modified and mutant mice. Cytogenetic and Genome Research 103 267-276. (doi:10.1159/000076812)

Drumond AL, Meistrich ML \& Chiarini-Garcia H 2011 Spermatogonial morphology and kinetics during testis development in mice: a highresolution light microscopy approach. Reproduction 142 145-155. (doi:10.1530/REP-10-0431)

Ehmcke J, Luetjens CM \& Schlatt S 2005 Clonal organization of proliferating spermatogonial stem cells in adult males of two species of non-human primates, Macaca mulatta and Callithrix jacchus. Biology of Reproduction 72 293-300. (doi:10.1095/biolreprod.104.033092)

Ikami K, Tokue M, Sugimoto R, Noda C, Kobayashi S, Hara K \& Yoshida S 2015 Hierarchical differentiation competence in response to retinoic acid ensures stem cell maintenance during mouse spermatogenesis. Development 142 1582-1592. (doi:10.1242/dev.118695)

Kluin PM \& de Rooij DG 1981 A comparison between the morphology and cell kinetics of gonocytes and adult type undifferentiated spermatogonia in the mouse. International Journal of Andrology 4 475-493. (doi:10.1111/j.1365-2605.1981.tb00732.x)

Leblond CP \& Clermont Y 1952 Definition of the stages of the cycle of the seminiferous epithelium in the rat. Annals of the New York Academy of Sciences 55 548-573. (doi:10.1111/j.1749-6632.1952.tb26576.x)

Matzuk MM \& Lamb DJ 2008 The biology of infertility: research advances and clinical challenges. Nature Medicine 14 1197-1213. (doi:10.1038/ nm.f.1895)

Meistrich ML \& Hess RA 2013 Assessment of spermatogenesis through staging of seminiferous tubules. Methods in Molecular Biology 927 299-307. (doi:10.1007/978-1-62703-038-0_27)

Nakata H, Wakayama T, Takai Y \& Iseki S 2015a Quantitative analysis of the cellular composition in seminiferous tubules in normal and genetically modified infertile mice. Journal of Histochemistry and Cytochemistry 63 99-113. (doi:10.1369/0022155414562045)

Nakata H, Wakayama T, Sonomura T, Honma S, Hatta T \& Iseki S $2015 \mathrm{~b}$ Three-dimensional structure of seminiferous tubules in the adult mouse. Journal of Anatomy 227 686-694. (doi:10.1111/joa.12375)

Nakata H, Wakayama T, Asano T, Nishiuchi T \& Iseki S 2017 Identification of sperm equatorial segment protein 1 in the acrosome as the primary binding target of peanut agglutinin (PNA) in the mouse testis. Histochemistry and Cell Biology 147 27-38. (doi:10.1007/s00418-0161478-8)

Nel-Themaat L, Vadakkan TJ, Wang Y, Dickinson ME, Akiyama H \& Behringer RR 2009 Morphometric analysis of testis cord formation in Sox9-EGFP mice. Developmental Dynamics 238 1100-1110. (doi:10.1002/dvdy.21954)

Oakberg EF 1956 A description of spermiogenesis in the mouse and its use in analysis of the cycle of the seminiferous epithelium and germ cell renewal. American Journal of Anatomy 99 391-414. (doi:10.1002/ aja.1000990303)

Russell LD, Ettlin RA, Sinha-Hikim AP \& Clegg ED 1990 Histological and Histopathological Evaluation of the Testis. Vienna, IL: Cache River Press.

Perey B, Clermont Y \& Leblond CP 1961 The wave of the seminiferous epithelium in the rat. American Journal of Anatomy 108 47-77. (doi:10.1002/aja.1001080105)

Received 27 June 2017

First decision 17 July 2017

Revised manuscript received 25 July 2017

Accepted 31 July 2017 